\title{
Research on the Ubiquitous Learning Model in the Context of Big Data
}

\author{
Jing Chang \\ Institute of Information Science and Technology, Guangdong University of Foreign Studies South \\ China Business College, Guangdong, Guangzhou 510545, China.
}

hapipingye@163.com

\begin{abstract}
In the age of big data, information resources are facilitating the construction of ubiquitous learning environment. By following the dynamically changing needs of learners and taking a new look at information service models, this paper starts with the connotation and characteristics of the information service model of ubiquitous learning, and then proposes to excavate from the massive educational data the information resources and learning partners that meet the needs of learners, recommend the learning activities that fit the cognitive style of learners, and provide self-adaptive, personalized education services for learners.
\end{abstract}

Keywords: Big data; ubiquitous learning; semantic aggregation; learning resources.

\section{Introduction}

Ubiquitous learning is a new learning style resulting from the interpenetration between computing technology and emerging education theories. It means that anyone can learn anytime and everywhere based on any device and situation with free access to any information and support required to complete learning tasks easily [1]. By its very nature, ubiquitous learning is the learning that centers on humans and focuses on learning tasks. In the ubiquitous learning environment, learning is a natural or spontaneous action. What learners are concerned about will be the learning tasks, rather than the learning tools or environmental factors.

Big data is a term for massive, complex and diverse data sets that are gathered by the data processing and application model based on cloud computing. Big data inject new potential into ubiquitous learning. It can store resulting data, record procedural data, interpret the macroscopic and overall state of education and more importantly it can better supervise the learning track and process of individuals and provide personalized services and guidance. At present, however, education information resources are disorderly increasing, weakly interoperable and loosely associated, which makes it impossible to provide one-stop learning services for learners.

By taking a new look at information service models and following the dynamically changing needs of learners, this paper proposes to excavate from the massive educational data the information resources and learning partners that meet the needs of learners, recommend the learning activities that fit the cognitive style of learners, and provide self-adaptive, personalized education services for learners.

\section{Elements and Connotation of the Information Services of Ubiquitous Learning}

Information services are information resources based activities in which all kinds of methods or technological means are adopted to collect, sort out, use information and to provide the relevant information products and services [2]. An information service model summarizes the elements of information service activities and the mutual relations between those elements [3]. People and machines (intelligent agents) integrate and work together as service subjects to blend into the ubiquitous learning situation, make use of wireless sensors to track learning process, clarify user needs through the interaction between interactive software and learners, design service modes and integrate information resources and learning environment, and provide adaptive services [4].

\subsection{Service Subjects.}

Service subjects must have powerful and flexible information retrieval ability, knowledge reasoning ability, intelligence analysis ability, and semantic aggregation ability. The responsibilities 
of service subjects are to perceive user needs flexibly, analyze and excavate meaningful knowledge on a real-time basis, and provide personalized, specialized services.

\subsection{Service Objects.}

Service objects refer to the people who need information services. As ubiquitous learning gets promoted, serve objects' learning situations are closely mingled with their living situations, the scope of their learning is expanded, and their learning needs are increasingly diversified and detailed, which jointly necessitate more accurate and more valuable information services. Following the maturity of Web 3.0 and other relevant technologies, users have a greater awareness and desire to create personalized information. Meanwhile, service objects can also get involved in the construction, communication and utilization of information resources, and create learning resources in collaboration with service subjects to promote the self-adaptive evolution of resources and the improved quality of information services.

\subsection{Service Methods.}

Service methods refer to the tools and means employed in the process of information services, and they are influenced by both learning style and technological level. The dynamic semantic association of resources is of great value because it greatly relieves learners from the pressure and burden of blindly searching resources, and functions as a "knowledge navigator" to help learners find, in a more direct manner, the resources they are interested in and need [5]. Information services emphasize the connectivity, situationality, and adaptability of services, and associate learning resources with networks to form an interconnected ubiquitous learning environment.

\subsection{Service Contents.}

Service contents refer to the products and services that are valuable to certain users. Full, real-time and personalized learning services are offered to smoothly advance the learning progress of learners and to facilitate the integration and innovation of knowledge. The service system tracks and records the learning process of learners to form learning portfolios, and offers stage-based, targeted service contents after clearly mastering the characteristics and needs of learners.

\section{Characteristics of the Information Service Model of Ubiquitous Learning}

The purpose of providing information services during ubiquitous learning is to satisfy the increasingly diversified explicit and implicit learning needs of service objects. The service model has the following characteristics:

\subsection{Intelligent Interaction.}

The age of big data is an age of intelligent services. Speaking from service contents, the information services of ubiquitous learning not only provide interdisciplinary information resources but more importantly offer collaborative learning partners from different subjects and promote the connection between learners' internal cognitive network and the external information network. Speaking from service subjects, intelligent expert system and specialized service team collaborate with and supplement each other to track the needs of different learners on a real-time basis, have in-depth interactions with learners, and provide learners with satisfactory services.

\subsection{Evolvability of Learning Resources.}

The learning resources in the ubiquitous learning environment are different from traditional learning objects in the way of organizing, management mode, basic characteristics, construction strategy, and retrieval and recommendation method. Ubiquitous learning resources attach greater importance to such features as semantic description, situational adaptation, intelligentization, and evolvable development. Yu Shengquan et al. point out that ubiquitous learning calls for more quantities of evolvable learning resources, and that the traditional static, solidified and slowly updated e-learning resources cannot meet the practical needs of ubiquitous learning [6]. Evolvability has become an important characteristic and tendency of future learning resources, which will pose a huge challenge to e-learning resources that are slowly updated and less motivated to evolve. Besides, the research on the evolution of learning resources will also become an important direction for studying future ubiquitous learning resources. 


\subsection{Adaptability of Situational Awareness.}

Situation is also called context. Context awareness can be used to support the interoperability and adaptability of learning facilities. Interoperability mainly means many different learning facilities are interoperable. For instance, the PDA, mobile phone, and PC used by learners can automatically synchronize, and are able to share the same address space and input/output ports. Adaptability refers to the fact that learning resources can adapt to learning facilities. For example, different user interfaces or learning contents can be provided according to the learning facilities currently available to learners.

\section{Conclusion}

Information technology plays a significant role in promoting the informationization of education. The information services of ubiquitous learning help learners excavate personalized knowledge from general information. Based on the needs of learners, such information services make use of big data analysis techniques to make specific learning resources, teaching methods and learning guidance available to learners, and inject new vitality into the differentiated development of individuals. Moreover, they will also continuously improve the quality of education information resources, enhance the utilization ratio of resources, and contribute to the better quality of information services. The development of computer and relevant technologies, improved communications technology, enhanced open network and computing power, and the emergence of more flexible software structure will all facilitate the realization and further perfection of the ubiquitous learning model.

\section{Acknowledgements}

"A Model Program for Cultivating the Applied Talents of Computer Science and Technology" under the 2015 Undergraduate Teaching Quality and Teaching Reform Project in Guangdong Province (Document No. [2015] 133 issued by the Department of Education of Guangdong Province concerning higher education)

\section{References}

[1] Yang Xianmin, Yu Shengquan. Design of Ubiquitous Learning Environment from the Ecological Perspective [J]. Educational Research, 2013, (3): 103-110.

[2] Zhang Shuhua, Wang Jingshan, Liu Luyin et al. Library Information Services in the Digital Age [M]. Beijing: Beijing Library Press, 2005.

[3] Liu Yuanyun, Li Zhimin. The Three Information Service Models of Modern Libraries [J]. Library Journal, 2013, (1): 26-31.

[4] Ding Jihong, Xiong Caiping et al. Research on the Information Service Model of Embedded Ubiquitous Learning in the Context of Big Data [J]. e-Education Research, 2015, 09, 30-35.

[5] [Austrian] Dietmar Jannach et al. Recommendation System [M]. Translated by Jiang Fan, Beijing: Posts \& Telecom Press, 2013: 8-9.

[6] Cheng Gang, Xu Jin, Yu Shengquan. New Development of the Standards of Learning Resources and Development Tendency of Learning Resources [J]. Journal of Distance Education, 2009, (4): 6-12. 